\title{
Monte-Carlo Simulations of Compton Polarization in Astrophysical Sources
}

\author{
Lenté Dreyer* \\ Centre for Space Research, North-West University, Potchefstroom, 2520, South Africa \\ E-mail: lentedreyer@gmail.com \\ Markus Böttcher ${ }^{\dagger}$ \\ Centre for Space Research, North-West University, Potchefstroom, 2520, South Africa \\ E-mail: Markus.Bottcher@nwu.ac.za
}

\begin{abstract}
The spectral energy distributions (SEDs) of astrophysical sources can be modeled with different radiation mechanisms. Polarization carries information on the astrophysical environment of astrophysical sources, such as the geometry and orientation of the magnetic field, the location of the emitting region, and the emission mechanism. Compton scattering can both produce or reduce the degree of polarization of the high energy photons. In this paper, a Monte-Carlo code - MAPPIES (Monte-Carlo Applications for Partially Polarised Inverse External-Compton Scattering) - is used to simulate the anisotropic Compton scattering off arbitrary thermal and non-thermal electrons, in order to study the high-energy polarization, and make predictions for various models (e.g. the Big Blue Bump feature in some blazar spectra and the prompt emission from $\gamma$-ray bursts (GRBs)).
\end{abstract}

7th Annual Conference on High Energy Astrophysics in Southern Africa - HEASA2019

28 - 30 August 2019

Swakopmund, Namibia

\footnotetext{
*Speaker.

$\dagger$ The work of Markus Böttcher is supported through the South African Research Chair Initiative (SARChI) of the South African National Research Foundation (NRF) and the Department of Science and Technology (DST), under SARChI Chair grant no. 64789. Disclaimer: Any opinion, finding and conclusion or recommendation expressed in this material is that of the authors and the NRF does not accept any liability in this regard.
} 


\section{Introduction}

The description of many high-energy astrophysical sources relies on the production of relativistic jets (e.g. active galactic nuclei (AGNs), $\gamma$-ray bursts (GRBs), and X-ray binaries) that are accompanied by the acceleration of particles up to very high energies and the production of secondary non-thermal radiation [1]. The radiation from these jet-like sources is characterized by their spectral energy distributions (SEDs) which can be modelled with different radiation mechanisms, whilst being consistent with the spectral shape of the SED (e.g. [2]). Discriminating between different models remains one of the main objectives in the field of high-energy astrophysics.

Polarization measurements of the high-energy (X-rays and $\gamma$-rays) radiation from astrophysical sources promise to deliver unambiguous constraints on the geometry and structure of the astrophysical environment. Compared to the orientation of the relativistic jet, polarization adds crucial information about the jet-physics and jet-formation models. Even though polarimetric measurement techniques remain less advanced for X-rays and $\gamma$-rays compared to the radio to optical regimes, there are future prospects of detecting the polarization in the X-ray and $\gamma$-ray regime from many sources (see e.g. [3, 4, 5] for reviews). Model predictions of the polarization for different radiation mechanisms are thus justified.

The general formalism for calculating the polarization of Compton radiation was developed by [6] in the Thomson regime, and applied specifically to synchrotron self Compton by [7]. Generally, the Klein Nishina cross-section is polarization dependent. Polarized photons scatter preferentially in the direction perpendicular to their electric field vector [8]. Polarization can therefore be induced when non-relativistic electrons scatter an anisotropic seed photon field, even if the seed photons are unpolarized. When the electrons are relativistic, the seed photon field is almost axisymmetric around the electron momentum in the electron rest frame, which makes any intrinsic anisotropy of the seed photons irrelevant. Compton scattering off relativistic electrons, therefore, does not induce polarization (e.g. [9]).

In a model where an unpolarized external radiation field scatters off an arbitrary thermal and non-thermal electron distribution, the ultraviolet/soft X-ray radiation results from scattering off thermal electrons, and is thus expected to be polarized. The hard X-rays and $\gamma$-rays result from scattering off relativistic electrons and are therefore not expected to be polarized. In this paper, a newly developed Monte-Carlo code - MAPPIES (Monte-Carlo Applications for Partially Polarised Inverse External-Compton Scattering) - is used to simulate Compton scattering off non-relativistic electrons (with both thermal and non-thermal energy distributions) in order to study the high-energy polarization from jet-like astrophysical sources.

\section{Compton scattering}

The MAPPIES code is applied to astrophysical sources that are associated with relativistic jets (e.g. jet dominated AGNs and GRBs). The emission region moves along the jet with a Lorentz factor $\Gamma_{j e t}=10$, assumed to be well localized and optically thin throughout the electromagnetic spectrum. The electrons are assumed to be non-relativistic (with thermal temperatures of $k T_{e}=$ $50 \mathrm{keV}$, and electron Lorentz factors of $\gamma \sim 1$ ) and isotropically distributed in the co-moving frame of the emission region. The electron energy (shown in Fig.1) is drawn from either a purely thermal 
(Maxwell) distribution or a hybrid (Maxwell + power-law) distribution. In the case of a hybrid electron energy distribution, a fraction $f_{n t h}=0.02$ of the electrons are assumed to be non-thermal, drawn from a power-law distribution, $n_{p l} \propto \gamma^{-p}$, with a power-law index of $p=2.5$ and a maximum electron Lorentz factor of $\gamma_{\max }=1.6 \times 10^{3}$. The seed photon field is assumed to unpolarized, isotropically distributed in the AGN rest frame, with energies drawn from a black body distribution (shown in Fig.2) with a single temperatures of $k T_{\text {rad }}=0.5 \mathrm{keV}$ (soft X-rays), $k T_{\text {rad }}=50 \mathrm{keV}$ (hard $\mathrm{X}$-rays), and $k T_{\text {rad }}=500 \mathrm{keV}$ ( $\gamma$-rays). ${ }^{1}$ In what follows, quantities in the electron rest frame and the AGN rest frame are denoted with subscripts $e$ and agn, respectively. All scattered photon properties are denoted with a superscript $s c$.

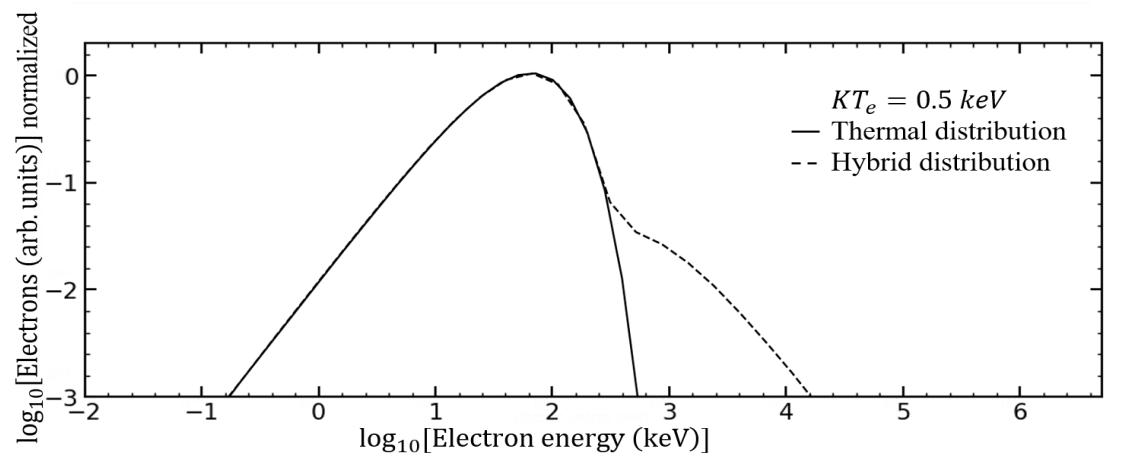

Figure 1: The energy distributions for non-relativistic $\left(k T_{e}=50 \mathrm{keV}\right)$ electrons drawn from either a purely thermal (Maxwell) distribution (solid curve) or a hybrid (power-law + Maxwell) distribution (dashed curve), with a fraction of $f_{n t h}=0.02$ electrons drawn from a power-law distribution with an index of $p=2.5$.

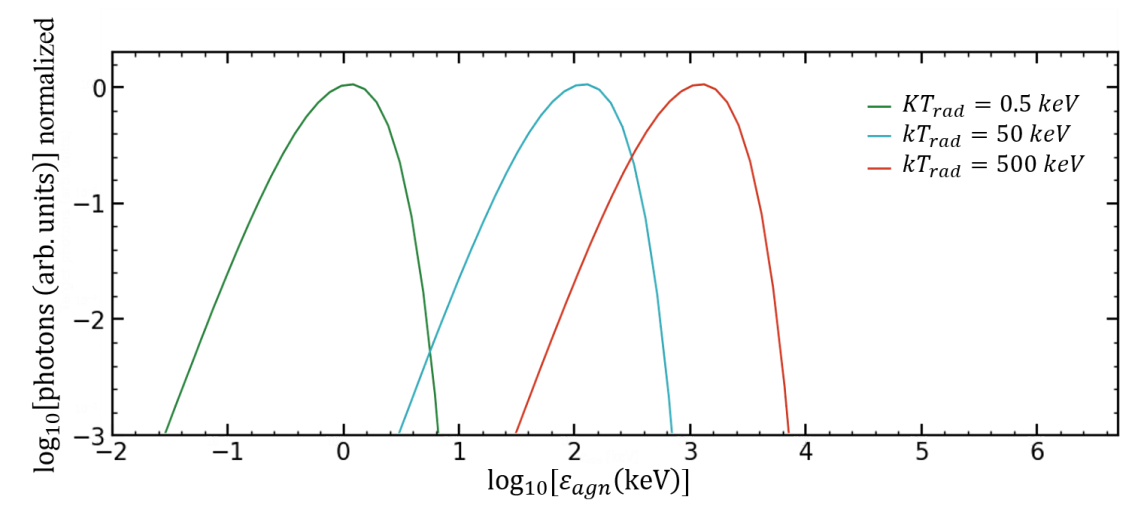

Figure 2: The seed photon energy distributions for soft X-rays $\left(k T_{\text {rad }}=0.5 \mathrm{keV}\right.$;green $)$, hard X-rays $\left(k T_{\text {rad }}=\right.$ $50 \mathrm{keV}$; blue $)$, and $\gamma$-rays $\left(k T_{\text {rad }}=500 \mathrm{keV}\right.$;orange $)$

The probability of a photon to scatter off an electron is determined by the Klein Nishina crosssection, most conveniently evaluated in the electron rest frame [1]. The polarization averaged

\footnotetext{
${ }^{1}$ The input parameters used in this study are arbitrary choices made for illustrative purposes and not based on a specific source or scenario. In the case of electrons drawn from a hybrid distribution, the fraction of non-thermal electrons $f_{n t h}=0.02$, as well as the power-law index of the power-law tail $p=2.5$ are values that can typically result from diffusive shock acceleration (e.g. [10]).
} 
differential Klein Nishina cross-section is given by

$$
\frac{d \sigma_{K N}}{d \Omega_{e}^{s c} d x_{e}^{s c}}=\frac{r_{e}^{2}}{2}\left(\frac{x_{e}^{s c}}{x_{e}}\right)^{2}\left(\frac{x_{e}^{s c}}{x_{e}}+\frac{x_{e}}{x_{e}^{s c}}-\sin ^{2} \Theta_{e}^{s c}\right) \delta\left(x_{e}^{s c}-\frac{x_{e}}{1+x_{e}\left[1-\cos \Theta_{e}^{s c}\right]}\right)
$$

where the $\delta$ function results from the energy and momentum conservation [1, 8]. In Equation 2.1, $x_{e}=\frac{\varepsilon_{e}}{m_{e} c^{2}}$ with $\varepsilon_{e}$ the energy of the seed photons, and $x_{e}^{s c}=\frac{\varepsilon_{e}^{s c}}{m_{e} c^{2}}$, with $\varepsilon_{e}^{s c}$ the scattered photon energy, $r_{0}$ is the classical electron radius, $\Theta_{e}^{s c}$ is the angle between the seed and the scattered photon (i.e., the polar scattering angle), and $d \Omega_{e}^{s c}=d \cos \Theta_{e}^{s c} d \Phi_{e}^{s c}$ is a solid angle element of the scattered photon's direction of motion. The dependence of the scattering cross-section on $x_{e}$ is given in Fig.3. The cross-section assumes a constant value at $x_{e} \ll 1$ (i.e., Thomson regime) and becomes smaller in the Klein Nishina regime where $x_{e} \gg 1$. Therefore, in the electron rest frame, the scattering is almost elastic in the Thomson regime with scattered energies $x_{e}^{s c} \sim x_{e}$. In the Klein Nishina regime, the energy exchange from the photon to the electron becomes substantial with $x_{e}^{s c} \sim 1$.

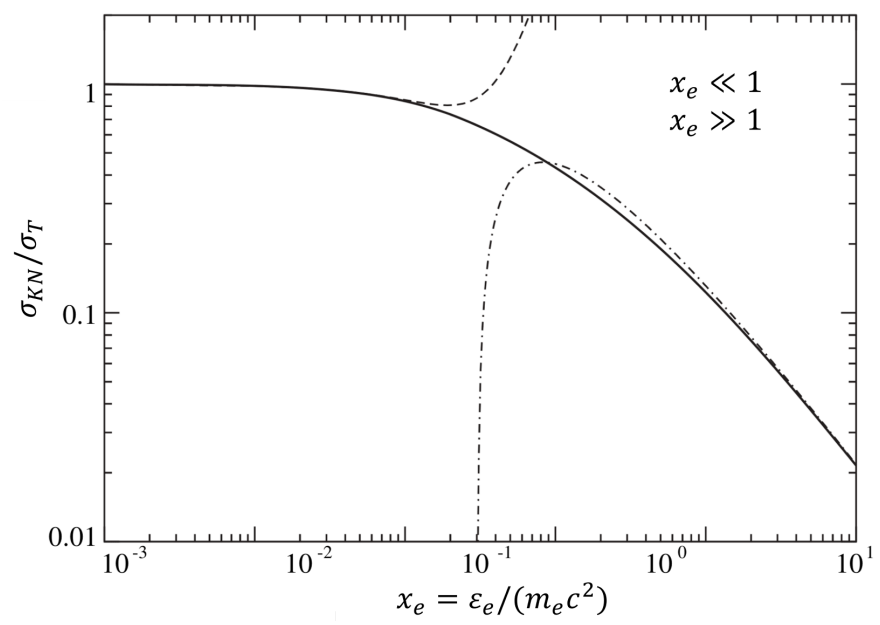

Figure 3: The Compton cross-section as a function of the dimensionless target photon-energy $x_{e}$ in the electron-rest frame. Also shown are the asymptotes for $x_{e} \ll 1$ (dashed), and $x_{e} \gg 1$ (dot-dashed). From [1].

Fig 4 shows the scattered photon energy distributions in the AGN rest frame. The seed photon energies are boosted with a factor of $\sim \Gamma_{j e t} k T_{\text {rad }}$ to the emission frame with the peak of the black body spectrum at $\sim 2.8\left(k T_{\text {rad }} / m_{e} c^{2}\right) \Gamma_{\text {jet }}$. Soft X-rays $\left(k T_{\text {rad }}=0.5\right.$; shown in green) are scattered in the Thomson regime with scattered photon energies $\varepsilon_{a g n}^{s c} \sim \Gamma_{j e t}^{2} \gamma^{2} \varepsilon_{a g n}$. Seed photons with $k T_{\text {rad }}=500 \mathrm{keV}$ (shown in orange) are scattered in the Klein Nishina regime with cut-off energies that correspond to the reduction of the cross-section in the electron rest frame. In the case of scattering off a non-thermal power-law distribution (shown with dashed lines in all figures discussed), Compton scattering in the Thomson regime also results in a power-law distribution of scattered photons.

\section{Compton Polarization}

The scattering cross-section is generally dependent on the polarization through the angular distri- 


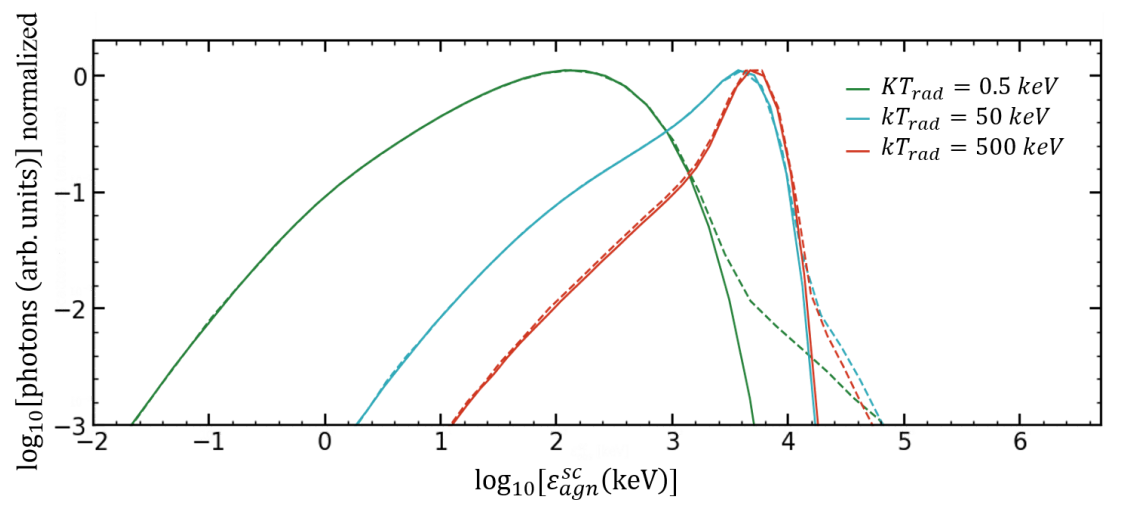

Figure 4: The scattered photon energy distributions for Compton scattering off electrons drawn from a thermal distribution (solid curves) and a hybrid (Maxwell + power-law) distribution (dashed curves).

bution of the photons, with

$$
\sigma_{K N} \propto \frac{x_{e}}{x_{e}^{s c}}+\frac{x_{e}^{s c}}{x_{e}}-2+4\left(\vec{e}_{e} \cdot \vec{e}_{e}^{s c}\right)
$$

where $\vec{e}_{e}$ and $\vec{e}_{e}^{s c}$ are the polarization vectors of the seed and scattered photons [8]. In the Thomson regime, the polarization term in Equation 3.1 dominates so that $\sigma_{K N} \propto 4\left(\vec{e}_{e} \cdot \vec{e}_{e}^{s c}\right)$ with $x_{e}^{s c} \sim x_{e}$. In the Klein Nishina regime, when $x_{e} \gg 1$, the polarization term becomes sub-dominant with $\sigma_{K N} \propto$ $\frac{x_{e}}{1}+\frac{1}{x_{e}}-2+4\left(\vec{e}_{e} \cdot \vec{e}_{e}^{s c}\right)$ since $x_{e}^{s c} \sim 1$. Therefore, polarization is expected to arise when photons are scattered in the Thomson regime while no polarization is expected to arise in the Klein Nishina regime. If the seed photons are unpolarized, the scattering process may produce linearly polarized photons, which produces an anisotropic azimuthal distribution of the scattered photons. In this section, the polarization signatures due to the Compton scattering effects described in the previous section will be discussed.

Following the treatment of Compton scattering of linearly polarized photons in Monte Carlo codes by [8], the contribution of every photon to the second $\left(Q_{i}^{s c}\right)$ and third $\left(U_{i}^{s c}\right)$ Stokes parameters is registered throughout the simulation (where the subscript $i$ refers to every individual photon). When the simulation is complete, $Q_{i}$ and $U_{i}$ are summed over all $N^{s c}$ scattered photons in the specified direction,

$$
Q^{s c}=\sum_{i=1}^{N^{s c}} Q_{i}^{s c} \quad \text { and } \quad U^{s c}=\sum_{i=1}^{N^{s c}} U_{i}^{s c}
$$

where $Q^{s c}$ and $U^{s c}$ are the Stokes parameters of the scattered radiation. The polarization degree (PD) and polarization angle (PA) may then be obtained with the Stokes formalism [11] where

$$
\mathrm{PD}=\frac{\sqrt{\left(Q^{s c}\right)^{2}+\left(U^{s c}\right)^{2}}}{N^{s c}} \quad \text { and } \quad \mathrm{PA}=\frac{1}{2} \arctan \frac{U^{s c}}{Q^{s c}}
$$

Fig.5 shows the PD (top panel), and PA (bottom panel) as a function of the scattered photon energy $\varepsilon_{a g n}^{s c}$ (left) and the viewing angle of the scattered photons $\Theta_{a g n}^{s c}$ (right). The PD of the scattered photons decreases with increasing photon energies: The values of the maximum PD are 
$\sim 30 \%$ for seed photon energies of $k T_{\text {rad }}=0.5 \mathrm{keV}$ (shown in green), $\sim 20 \%$ for $k T_{\text {rad }}=50 \mathrm{keV}$ (shown in blue), and $\sim 5 \% k T_{\text {rad }}=500 \mathrm{keV}$ (shown in orange). Since the orientation of the polarization signatures does not change significantly for different electron distributions, the PD is similar for Compton scattering off a non-thermal, power-law distribution of electrons (dashed curves) to those of a purely thermal distribution (solid curves). The maximum PD is at viewing angles of $\Theta_{\text {agn }}^{s c} \sim \Gamma_{j e t}^{-1}$ rad (shown with a grey line) in the AGN rest frame that corresponds to the edge-on view with respect to the jet.

The PD as a function of $\varepsilon_{\text {agn }}^{s c}$ can be used to evaluate which energy band provides the most promising prospect for the detection of high-energy polarization from the source. For the photons that are scattered in the Thomson regime, the maximum PDs occurs where the non-relativistic electrons scatter the seed photons (to an energy of $\Gamma_{j e t}^{2} k T_{\text {rad }}$ ). The maximum PD lies within the energy regime of $\sim 10-10^{3} \mathrm{keV}$ for $k T_{\text {rad }}=0.5 \mathrm{keV}$ (shown in green), $\sim 10^{2}-10^{4} \mathrm{keV}$ for $k T_{\text {rad }}=50 \mathrm{keV}$ (shown in blue), and $\sim 10^{3}-10^{4} \mathrm{keV}$ for $k T_{\text {rad }}=500 \mathrm{keV}$ (shown in orange). In the case of Compton scattering of electrons drawn from a non-thermal power-law distribution (dashed curves), more photons are available in higher energy regimes than in the case of purely thermal electrons (solid curves). However, these photons are unpolarized since the non-thermal electrons are relativistic. Regardless of the electron distribution, the PA (shown in bottom panels of Fig.5) for the unpolarized radiation is undefined, and randomly assumes all possible values for PA $\in[0, \pi] \mathrm{rad}$. For the fraction of the scattered photons that is polarized, the PA assumes a constant value of $\sim \frac{\pi}{2}$ rad corresponding polarization perpendicular with respect to the jet.

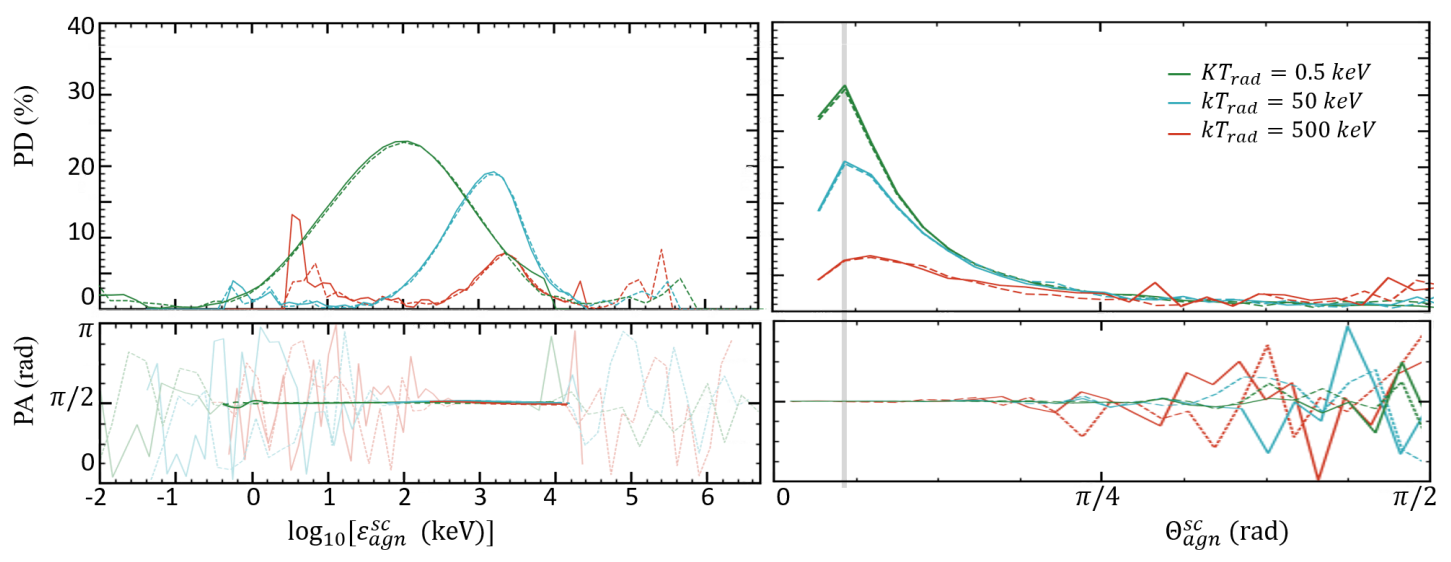

Figure 5: The PD (top panels) and PA (bottom panels) as a function of the scattered photon energy (left panel) and viewing angle (right panel).

\section{Conclusion}

The effects of Compton scattering between photons and electrons are dependent on the seed photon energy, the Lorentz factor of the electrons $\gamma$ (thus the thermal temperatures and the energy distribution of the electrons) as well as the bulk Lorentz factor $\Gamma_{j e t}$ of the emission region. The PD of the scattered photons depends, in turn, on the effects of the Compton scattering, due to the polarization dependence of the Klein Nishina cross-section. The PD decreases with increasing photon 
energies, thus implying that polarization will be expected to arise in Thomson regime, while no polarization is expected to be induced for photons scattered in the Klein Nishina regime. The energy regime of the polarized Compton emission shifts to higher energies and becomes smaller for higher seed photon energies. The PD is at its maximum for viewing angles perpendicular to the jet in the emission-region rest frame that correspond to angles of $\sim \Gamma_{j e t}^{-1} \mathrm{rad}$ in the AGN rest frame. The PA that corresponds to the polarized photons has a constant value of $\mathrm{PA}=\frac{\pi}{2} \mathrm{rad}$, regardless of the photon energy or the electron distribution.

The MAPPIES code used in this paper is capable of predicting the Compton polarization in different jet-like astrophysical sources for different photon energies and electron distributions. Future applications of the code include the simulation of the polarization signatures from a model where the soft X-ray excess (Big Blue Bump) in blazar spectra arises from a bulk Compton process (Compton scattering of an external field by thermal electrons), as proposed in e.g. [12] for the BL Lac object AO 0235+164.

\section{References}

[1] M. Böttcher D. E. Harris \& H. Krawczynski, Relativistic Jets from Active Galactic Nuclei, Wiley, Berlin 2012.

[2] M. Böttcher, A. Reimer, K. Sweeney, \& A. Prakash, Leptonic and hadronic modeling of Fermi-detected blazars., ApJ 768(1) (2013) 54.

[3] H. Krawczynski, A. Garson III, Q. Guo, M. G. Baring. et al. Scientific prospects for hard X-ray polarimetry, Astroparticle Physics 34(7) (2011) 550.

[4] S. Fabiani, Instrumentation and future missions in the upcoming era of X-ray polarimetry, Galaxies 6(2) (2018) 54.

[5] M. L. McConnell \& J. M. Ryan, Status and prospects for polarimetry in high energy astrophysics, New Astronomy Reviews 48 (2004) 215.

[6] S. Bonometto, S. Cazzola \& A. Saggion, Polarization in Inverse Compton Effect, A\&A 7 (1970) 292.

[7] S. Bonometto \& A. Saggion, Polarization in inverse Compton scattering of synchrotron radiation., A\&A 23 (1973) 9.

[8] G. Matt, M. Feroci, M. Rapisarda \& E. Costa, Treatment of Compton scattering of linearly polarized photons in Monte Carlo codes, Radiat. Phys. Chem. 48(4) (1996) 403.

[9] H. Krawczynski, The Polarization Properties of Inverse Compton Emission and Implications for Blazar Observations with the GEMS X-Ray Polarimeter, ApJ 744(1) (2012) 30.

[10] E. J. Summerlin, M.G. Baring, Diffusive acceleration of particles at oblique, relativistic, magnetohydrodynamic shocks., ApJ 745(1) (2012) 63.

[11] G. G. Stokes, On the Composition and Resolution of Streams of Polarized Light from different Sources, Trans. Cambridge Philos. 9 (1851) 399.

[12] M.G. Baring, M. Böttcher \& E. J. Summerlin. Probing acceleration and turbulence at relativistic shocks in blazar jets., MNRAS 464(4) (2017) 4875. 